\title{
Editorial
}

\section{Functionalized Polymeric Materials for Catalytic Upgrading of Biobased Feedstocks}

\author{
Heng Zhang $\mathbb{D}^{1}$, Chunbao Charles $X u \mathbb{D}^{2},{ }^{2}$ and Song Yang $\mathbb{D}^{1}$ \\ ${ }^{1}$ State Key Laboratory Breeding Base of Green Pesticide \& Agricultural Bioengineering, Key Laboratory of Green Pesticide \& \\ Agricultural Bioengineering, Ministry of Education, State-Local Joint Laboratory for Comprehensive Utilization of Biomass, \\ Center for Research \& Development of Fine Chemicals, Guizhou University, Guiyang, Guizhou 550025, China \\ ${ }^{2}$ Institute for Chemicals and Fuels from Alternative Resources (ICFAR), Department of Chemical and Biochemical Engineering, \\ Western University, London, Ontario, Canada N6A $5 B 9$
}

Correspondence should be addressed to Heng Zhang; hzhang23@gzu.edu.cn, Chunbao Charles Xu; cxu6@uwo.ca, and Song Yang; hzx.msm@gmail.com

Received 2 March 2021; Accepted 3 March 2021; Published 19 March 2021

Copyright (c) 2021 Heng Zhang et al. This is an open access article distributed under the Creative Commons Attribution License, which permits unrestricted use, distribution, and reproduction in any medium, provided the original work is properly cited.

Modern life depends on polymers, from materials applied in the manufacture of clothing, houses, cars, and airplanes to those demonstrating complex adhibitions in medicine, diagnostics, and electronics. More importantly, both natural and synthetic polymeric materials bearing versatile functionalities are gaining research interest for applications in catalysis, especially in the catalytic valorization of renewable biomass feedstocks (the abundant, natural, and low-cost biopolymers on the earth) to value-added chemicals and liquid biofuels [1-3]. As an important class of heterogeneous catalysts, functionalized polymeric materials with tailorable and attractive catalytic properties exhibit dramatic improvement of catalytic activity and selectivity in biomass refinery to produce energy and chemicals, compared with conventional counterparts.

The Special Issue "Functionalized Polymeric Materials for Catalytic Upgrading of Biobased Feedstocks" focuses on the introduction of polymer/polymeric catalysts preparation, functionalization of polymeric materials, and applications to guide the development and application of functionalized polymeric materials in biomass transformation. Five eminent research groups in the field of polymeric materials and biomass valorization have kindly accepted our invitation to participate in this special issue, briefly listed as follows:

In the paper entitled "Progress of Catalytic Valorization of Bio-Glycerol with Urea into Glycerol Carbonate as a Monomer for Polymeric Materials," Zhang et al. summarized the state-of-the-art accomplishments made in the efficient transformation of bioglycerol and urea into glycerol carbonate as a polymer monomer. Emphasis was given to the catalytic performance of the relevant catalysts such as zinc, magnesium, tungsten, ionic liquid-based catalysts, and waste-derived materials, reaction conditions, and possible pathways. Besides, how to modify the reaction conditions that will influence the related activities and selectivity to glycerol carbonate was also depicted [4].

In the paper entitled "Functionalized Metal-Organic Framework Catalysts for Sustainable Biomass Valorization," Liu et al. depicted metal-organic frameworks (MOFs), also identified as micro- and mesoporous coordination polymers (PCPs) to be employed as versatile heterogeneous polymeric catalysts for biomass upgrading. They highlighted recently developed four types of MOFs like pristine MOFs and their composites, MOF-supported metal nanoparticles, acid-functionalized MOFs, and biofunctionalized MOFs for the production of green, sustainable, and industrially acceptable biomass-derived platform molecules: (1) upgrading of saccharides, (2) upgrading of furan derivatives, and (3) upgrading of other biobased compounds [5].

In the paper entitled "Efficient Production of Methyl Oleate Using a Biomass-Based Solid Polymeric Catalyst with High Acid Density," Wang et al. investigated the biomassbased chitosan polymer acid catalyst $\left(\mathrm{CS}-\mathrm{SO}_{3} \mathrm{H}\right)$ for biodiesel production. The results showed that $\mathrm{CS}-\mathrm{SO}_{3} \mathrm{H}$ morphology 
exhibited a sphere of about $10 \mu \mathrm{m}$ diameter, and the acid density was as high as $3.81 \mathrm{mmol} / \mathrm{g}$. The catalyst demonstrated good catalytic activity in the esterification of oleic acid and methanol, which is a model reaction of the preesterification process in the preparation of biodiesel from feedstocks with high acid values. Under the optimum reaction conditions (15/1 methanol/oleic acid mole ratio and $3 \mathrm{wt} \%$ catalyst dosage at $75^{\circ} \mathrm{C}$ for $3 \mathrm{~h}$ ), the yield of methyl oleate came up to $95.7 \%$. After four times of reuse, the yield of the catalyst could still reach $85.7 \%$, which indicated that the catalyst depicted good catalytic activity and stability and showed potential application prospects [6].

In the paper entitled "Adsorption of Chelerythrine from Toddalia asiatica (L.) Lam. by ZSM-5," Liu et al. performed a study focusing on the separation and purification of active components from biomass by using the micropolymer catalyst zeolite molecular sieves ZSM-5. Batch experiments revealed that the adsorption efficiency was affected by the solution $\mathrm{pH}$, and the optimum initial solution $\mathrm{pH}$ was 6 . The maximum adsorption capacity of CHE was found at a solid-liquid ratio of $2: 15$. This material was thought to be a promising adsorbent for purifying alkaloids (especially quaternary ammonium alkaloids) from medicinal herbs. In addition, a technique for isolating active components from the pretreatment process of biomass was also provided [7].

In the paper entitled "3-Bromopyridine-Heterogenized Phosphotungstic Acid for Efficient Trimerization of BiomassDerived 5-Hydroxymethylfurfural with 2-Methylfuran to $\mathrm{C}_{21}$ Fuel Precursor," Xu et al. employed the halogenated pyridineheterogenized HPW as a heterogeneous acidic catalyst for the conversion of biomass-derived 5-hydroxymethylfurfural (HMF) and 2-methylfuran (2-MF) to $\mathrm{C}_{21}$ fuel precursor (MMBM). Single-factor optimization was utilized to test the catalytic performance of 3-BrPyPW; a good MMBM yield of $57.1 \%$ with $82.0 \%$ 2-MF conversion could be obtained under optimum reaction conditions. The $3-\mathrm{BrPyPW}$ catalyst could be reused for four cycles without a significant decrease in its activity, which was characterized without obvious structure change after recycles. Moreover, a dominant reaction pathway for the synthesis of MMBM from HMF and 2-MF was elucidated [8].

As Guest Editors, we would like to appreciate all authors for their valuable contributions and the referees for their excellent work in reviewing the submitted manuscripts.

\section{Conflicts of Interest}

The editors declare that there is no conflict of interest regarding the publication.

Heng Zhang
Chunbao Charles Xu
Song Yang

\section{References}

[1] H. Zhang, H. Li, C. Xu, and S. Yang, "Heterogeneously chemo/enzyme-functionalized porous polymeric catalysts of high- performance for efficient biodiesel production," ACS Catalysis, vol. 9, no. 12, pp. 10990-11029, 2019.

[2] H. Zhang, H. Li, Y. Hu, K. T. Venkateswara Rao, C. (. C.). Xu, and S. Yang, "Advances in production of bio-based ester fuels with heterogeneous bifunctional catalysts," Renewable and Sustainable Energy Reviews, vol. 114, article 109296, 2019.

[3] H. Li, Z. Fang, R. L. Smith, and S. Yang, "Efficient valorization of biomass to biofuels with bifunctional solid catalytic materials," Progress in Energy and Combustion Science, vol. 55, pp. 98194, 2016.

[4] H. Zhang, H. Li, A. Wang, C. Xu, and S. Yang, "Progress of catalytic valorization of bio-glycerol with urea into glycerol carbonate as a monomer for polymeric materials," Advances in Polymer Technology, vol. 2020, Article ID 7207068, 17 pages, 2020.

[5] X. Liu, Z. Liu, and R. Wang, "Functionalized metal-organic framework catalysts for sustainable biomass valorization," Advances in Polymer Technology, vol. 2020, Article ID 1201923, 11 pages, 2020.

[6] A. Wang, H. Zhang, H. Li, and S. Yang, "Efficient production of methyl oleate using a biomass-based solid polymeric catalyst with high acid density," Advances in Polymer Technology, vol. 2019, no. 11, Article ID 4041631, 11 pages, 2019.

[7] Y. Liu, J. Guo, Z. Xiao, D. Peng, and K. Song, "Adsorption of chelerythrine from Toddalia asiatica (L.) Lam. by ZSM-5," Advances in Polymer Technology, vol. 2020, Article ID 9408921, 9 pages, 2020.

[8] Y. Xu, Z. Yu, H. Li, and S. Yang, "3-bromopyridine-heterogenized phosphotungstic acid for efficient trimerization of biomass-derived 5-hydroxymethylfurfural with 2-methylfuran to $\mathrm{C}_{21}$ fuel precursor," Advances in Polymer Technology, vol. 2020, no. 12, Article ID 6438490, 12 pages, 2020. 